\title{
DESENVOLVIMENTO DE UM WEBSITE EDUCACIONAL PARA O ENSINO DO PROCESSO DE ENFERMAGEM EM CARDIOLOGIA
}

Francisco Railson Bispo de Barros¹, Claudevan Viana Amâncio², Márcia Danielle da Silva Ferreira ${ }^{3}$

Objetivo: descrever o processo de criação de um Website educacional para acadêmicos e profissionais enfermeiros contendo informações sobre o Processo de Enfermagem em Cardiologia. Metodologia: pesquisa experimental descritiva, baseada no modelo teórico de Tronchim para a construção de Website, composta das fases de conceitualização, desenvolvimento, implementação e avaliação. Resultados: o site possui 50 páginas contendo informações, orientações, fotos e ilustrações, estruturadas em tópicos com os conceitos básicos da anatomia e fisiologia cardíaca, coleta de dados, diagnósticos de enfermagem, planejamento, implementação e avaliação. Conclusão: a criação e desenvolvimento do Website foram descritos, podendo ser acessados no www.webcardio.net.br, na opção pesquisa.

Descritores: Internet, Ensino, Processos de Enfermagem, Cardiologia.

\section{DEVELOPMENT OF AN EDUCATIONAL WEBSITE TO TEACH CARDIOLOGY IN THE NURSING PROCESS}

Objective: To describe the process of creating an educational website for nursing and professional academics with information on the Nursing Process in Cardiology. Methodology: descriptive experimental research, based on the theoretical model of Tronchim to build websites, consisting of stages of conceptualization, development, implementation and evaluation. Results: The site has 50 pages containing information, directions, photos and illustrations, structured topics with the basics of anatomy and cardiac physiology, data collection, nursing diagnosis, planning, implementation and evaluation. Conclusion: the creation and development of the website have been described and can be accessed at www.webcardio.net.br the search option.

Descriptors: Internet, Education, Nursing Process, Cardiology.

\section{DESARROLLO DE UN SITIO WEB EDUCATIVO PARA LA ENSEÑANZA DEL PROCESO DE ENFERMERÍA EN CARDIOLOGÍA}

Objetivo: Describir el proceso de creación de un sitio web educativo para enfermeras académicos y profesionales con información sobre el Proceso de Enfermería en Cardiología. Metodología: la investigación experimental descriptivo, basado en el modelo teórico de Tronchim para construir sitios web, que consiste en las etapas de conceptualización, desarrollo, implementación y evaluación. Resultados: el sitio tiene 50 páginas que contienen información, direcciones, fotos e ilustraciones, temas estructurados con los fundamentos de la anatomía y la fisiología cardiaca, la recopilación de datos , diagnóstico de enfermería, planificación, ejecución y evaluación. Conclusión: la creación y desarrollo de la página web se han descrito y se puede acceder en www.webcardio.net.br la opción de búsqueda.

Descriptores: Internet, Educación, Proceso de Enfermería, Cardiología.

${ }^{1}$ Enfermeiro. Especialista em Cardiologia e Hemodinâmica. Fundação de Medicina Tropical. Email:raylsonbarros@hotmail.com ${ }^{2}$ Enfermeiro. Especialista em UTI. Centro Universitário do Norte - UNINORTE/Laureate.

${ }^{3}$ Enfermeira. Mestre em Ciências Aplicadas à Hematologia. UNINORTE/Laurete. 


\section{INTRODUÇÃO}

A aprendizagem do processo de enfermagem tem sido uma preocupação constante no cenário da enfermagem, uma vez que é um método amplamente aceito e tem sido sugerido como um método científico para orientar e qualificar a assistência de enfermagem. $O$ processo tem sido definido como forma sistemática e dinâmica de prestar cuidados de enfermagem, sendo realizado por meio de cinco etapas interligadas, começando pela coleta de dados, seguido de avaliação, diagnóstico, planejamento, implementação e avaliação(1).

De acordo com as normas americanas e canadenses, a prática de enfermagem exige a utilização eficiente do processo de enfermagem e participação dos profissionais em atividades que contribuam para o desenvolvimento permanente de conhecimentos sobre essa metodologia. $\mathrm{Na}$ prática, porém, nem todas as etapas são sistematicamente aplicadas, sendo que estudos têm revelado dificuldades no estabelecimento e na utilização do processo de enfermagem nas instituições durante os últimos anos, especialmente no Brasil(1-2).

Estudos mostram que o processo de enfermagem apresenta fragilidade em sua aplicabilidade quando direcionado aos portadores de doenças cardiovasculares, seja por fatores estruturais do ambiente de trabalho, déficits do conhecimento do próprio processo como um todo ou em partes, e até mesmo da anatomia, fisiologia e patologias do sistema cardiovascular. Para que se pense em melhorias desse quadro, aluno e profissional necessitam atualizarem-se no âmbito teórico-prático, utilizando, entre outros meios, a internet, que se tornou um dos mais importantes veículos de transmissão de informações ${ }^{(3)}$.

Ensinar alunos e profissionais enfermeiros e mantê-los atualizados têm sido grandes desafios no meio acadêmico e hospitalar, que tem encontrado no ambiente digital de aprendizagem uma importante ferramenta de apoio. A divulgação de documentos eletrônicos e sites na web ganha espaço, destacando-se esses como fontes virtuais de hipertexto que funcionam como recursos para estudos e veículos de divulgação de informações, colocando o aluno ou profissional no controle do processo de aprendizagem ${ }^{(4-5)}$.

O desenvolvimento de um Website voltado para o ensino do processo de enfermagem pode ser classificado como uma proposta inovadora de educação que visa atualizar o profissional enfermeiro, aproximar a teoria da prática, bem como a criação e a manutenção de processos assistenciais administrativos que garantam a qualidade do atendimento e a segurança do paciente, haja vista que é notória a fragilidade do modelo tradicional de ensino na construção de um saber mediano no protagonismo do aluno(6). Portanto, este estudo teve como objetivo descrever um modelo de desenvolvimento de um Website educacional sobre o processo de enfermagem em Cardiologia.

\section{METODOLOGIA}

Este trabalho constitui-se em uma pesquisa experimental descritiva. Para a evolução do estudo, foi adotado o modelo geral de criação de Websites sugerido por Trochim. Esse modelo envolve quatro grandes fases que são: conceituação; desenvolvimento; implementação e avaliação ${ }^{(7-9)}$

\section{Fase 1: Conceituação}

Nessa fase determinase o público-alvo, o objetivo, a análise e a definição do conteúdo que será abordado(8) Este estudo foi direcionado a acadêmicos e profissionais da área de enfermagem. Para a identificação dos artigos que constituíram o referencial teórico do Website, realizou-se, em agosto de 2015, uma busca nas bases de dados MEDLINE, LILACS, com descritores em português e inglês, tais como: Internet (Internet), Ensino (Teaching), Educação (Education), Processos de Enfermagem (Nursing Process), Cardiologia (Cardiology), procurando por publicações na temática escolhida.

Após a reunião dos artigos e materiais de interesse para a elaboração do Website, foi feita a definição do conteúdo que contemplaria os objetivos previamente definidos para que as informações providenciassem o conhecimento necessário, capaz de auxiliar no processo ensino-aprendizagem e na qualidade sistemática da assistência de enfermagem.

\section{Fase 2: Desenvolvimento}

Essa fase diz respeito à criação, estruturação e teste do website. Nessa fase, foram escolhidos o logotipo, o layout, a cor, a forma de apresentação e os links ${ }^{(10)}$. Para a sua criação, foram necessários conhecimentos gerais de informática, 
edição de páginas web, além de recursos materiais, tais como: recursos de hardware, periféricos e recursos de software.

Antes da construção das páginas web, as mídias (texto, imagens e animações) que fizeram parte do conteúdo foram previamente preparadas com o uso de editor de texto, digitalizador (scanner) e editor de imagens. Para a construção das páginas web, foi utilizado como plataforma um software utilizado para criação/edição e exibição de apresentações gráficas (PowerPoint 2007 da Microsotf Corporation ()), evitando-se maior gasto de tempo com a escrita integral por códigos na linguagem HyperText Markup Language (HTML)(10).

O Website foi desenvolvido em um computador com o sistema operacional Windows 8.1 da Microsoft Corporation $®$. Para a criação do hiperdocumento, foi escolhido o programa Word do pacote Office 2007 da Microsoft Corporation ®, pela sua facilidade e sua flexibilidade com o editor de página web adotado $^{(10)}$

Como editor de página da web, foi selecionado o software Dreamweaver MX da Macromedia ${ }^{\circledR}$, que é um editor visual profissional que cria e gerencia sites e páginas da web. Com esse programa, é fácil criar páginas compativeis com diversos navegadores e plataformas. O Dreamweaver MX® oferece ferramentas avançadas de desenho e layout, bem como facilita a utilização dos recursos do HTML dinâmico, como os comportamentos e camadas ${ }^{(10-11)}$

\section{Fase 3: Implementação}

Essa fase envolveu a publicação do Website na rede mundial de computadores. Assim, são descritos os processos de registro de domínio, contratação dos serviços de um provedor comercial, publicação e a atualização das informações a serem disponibilizadas ${ }^{(11)}$.

\section{Fase 4: Avaliação}

Após a publicação do Website na rede mundial de computadores, avaliou-se seu desempenho estrutural, ou seja, velocidade de busca, abertura de páginas e subsequentes, navegação nos frameset e interligação com os links referências do conteúdo abordado, subsidiando maior explanação do assunto proposto e treinamento do olhar clínico com estudos de caso(7).

\section{RESULTADOS}

Devido à temática do Processo de Enfermagem em Cardiologia, o título colocado no Website foi WebCardio; "Web", referindo-se à palavra "Web site" e "Cardio", da palavra "coração". A construção do WebCardio resultou em um hiperdocumento composto por 50 páginas, sendo uma para a página inicial, 44 distribuídas em oito seções do conteúdo temático e cinco para apresentação do site, termos e condições de uso, mapa do site, página do facebook e referências. O tempo total gasto na construção do site foi, aproximadamente, de quatro meses.

$\mathrm{O}$ acesso ao Website deve ser feito pelo endereço: www. webcardio.net.br, na opção pesquisa. A página inicial (home) foi elaborada dentro de um frameset duplo que auxilia na navegação de todo o site constituído de duas páginas:

1. topo.htm, contendo o banner personalizado para o website, englobando o logotipo do WebCardio.

2. menu.htm, contendo dez opções de menu, que foram criados com o Adobe Dreamweaver MX (arquivos gerados em páginas HTML [Hypertext Markup Language]);

$\mathrm{Na}$ definição do frameset, o conjunto dessas duas páginas, formou a página index.htm, ou seja, a página inicial do website. A partir da página inicial, o usuário tem acesso a todo o conteúdo do site por meio do frame lateral esquerdo, contendo dez opções de menu.

\section{Descrição dos principais conteúdos do WebCardio}

Na página inicial Index, no topo está contido o logotipo do WebCardio. Por intermédio do frameset lateral esquerdo, está disponibilizado o menu, onde o usuário inicia o acesso ao conteúdo didático-científico do site. Nessa página, no frame superior, estão apresentados um link para os termos e condições de uso e um link para o mapa do site. No frame direito, encontram-se os links da Sociedade Brasileira de Cardiologia, Sociedade Brasileira de Arritmia Cardíaca, American Heart Association, e European Society of Cardiology

No frame lateral esquerdo, estão disponibilizadas dez opções de menu, assim distribuídas: O que é o WebCardio: Anatomia do Coração; Fisiologia do Coração; Coleta de Dados: Diagnósticos de Enfermagem; Planejamento; Implementação; Avaliação; Eletrocardiograma; Referências. Os hiperlinks do site serão apresentados a seguir.

Selecionando a opção $O$ que é o web site no frameset lateral esquerdo, abre a página contendo informações sobre o autor, a finalidade do estudo, o conteúdo do website, os objetivos das informações disponibilizadas, o público alvo e indicações para a leitura dos termos e condições de uso do website antes de acessar o conteúdo do mesmo.

No link Anatomia do Coração, o usuário poderá visualizar o conteúdo sobre noções básicas das estruturas que compõem o coração, assim como o conceito do mesmo e termos da área cardiológica. Encontram-se no rodapé os hiperlinks: parede cardiaca, átrios, ventrículos, valvas, coronárias, sistema de condução, fontes e referências.

A opção Fisiologia do Coração tem por função apresentar a funcionalidade da bomba cardíaca, do relaxamento (diástole) à contração (sístole) e o percurso do sangue venoso e arterial. Descendo a barra de rolagem, o usuário encontrará hiperlinks 
no rodapé com as seguintes informações: circulação sistêmica, circulação pulmonar e fontes e referências.

A primeira etapa do Processo de Enfermagem está no link Coleta de Dados, correspondente ao levantamento de dados do método científico. Os hiperlinks no rodapé correspondem às seguintes informações: identificação, doença e tratamento, exame físico, aspectos psicossociais, fontes e referências.

No link Diagnóstico de Enfermagem, o usuário poderá visualizar a segunda etapa do PE que objetiva apresentar a padronização da linguagem de enfermagem fundamentada na Taxonomia da North American Nursing Diagnosis Association (NANDA), a forma de montagem e trabalhos realizados no Brasil sobre o perfil diagnóstico dos pacientes cardiopatas. Os hiperlinks no rodapé correspondem às seguintes informações: título, fatores relacionados, características definidoras, fatores de risco, definição, Diagnósticos de Enfermagem (DE) de cardiopatas, fontes e referências.

A opção Planejamento apresenta a terceira etapa do $\mathrm{PE}$, onde o usuário aprende a estabelecer as prioridades para os problemas diagnósticos, a fixação de resultados, o registro escrito de $D E$, dos resultados esperados e das prescrições de enfermagem de modo organizado. Os hiperlinks no rodapé correspondem às seguintes informações: prioridades, resultados, exemplo, fontes e referências.

No link Implementação, quarta etapa do PE, estabelecida as prioridades dos diagnósticos na etapa de planejamento, são apresentados os critérios para prescrição dos cuidados de enfermagem, assim como, definição de quem vai fazer o que e quando fazer.

Por último, no link Avaliação, é apresentada a definição e a importância de se avaliar o que foi feito, assim como os critérios a serem adotados, sejam eles para substituição ou manutenção do plano de cuidados. Estabelecido diagnóstico, planejamento e implementação. É nessa etapa que será definido se os critérios estabelecidos foram satisfatórios ou não.

O link Eletrocardiograma tem a função de apresentar os princípios básicos de sua funcionalidade, traçado eletrocardiográfico normal e critérios de avaliação e interpretação. Os hiperlinks no rodapé correspondem às seguintes informações: derivações, derivações periféricas, derivações precordiais, papel milimetrado, ondas e complexo, segmentos e intervalos, analise do ECG e fontes e referências.

Na opção Referências é apresentado o referencial teórico consultado para cada tema. Vale ressaltar que em cada hiperlink, no rodapé, constam o link das fontes e referências, objetivando a consulta imediata do mesmo caso o usuário queira se aprofundar no tema abordado em questão.

As páginas Termos e condições de uso e Mapa do site têm o propósito de respaldar o autor no que diz respeito ao conteúdo, finalidade e objetivos do Website e guiar o usuário na navegação dos hiperlinks respectivamente. Uma vez clicado no link de escolha no mapa do site, o usuário será automaticamente direcionado ao conteúdo do mesmo.

\section{DISCUSSÃO}

Considera-se que o ambiente digital abre espaço para integrar diferentes teorias de aprendizagem, colocandoas lado a lado, complementando e aproveitando, o que há de melhor em cada uma delas, além de favorecer o processo ensino-aprendizado. Vários teóricos são citados e utilizados como referencial no desenvolvimento de ferramentas educacionais para o ambiente virtual, porém nenhum deles encerra em si as possibilidades que o ambiente abre ao processo ensino aprendizagem.

Dessa forma, observa-se que a Internet e o ambiente digital de aprendizagem, constituem-se em uma poderosa ferramenta para o apoio do processo de ensino-aprendizagem. Seu uso crescente na área do ensino à saúde vem sendo avaliado como um importante facilitador, por acrescentar significado e concretude aos conteúdos que precisam ser aprendidos e que são abstratos e impossiveis de serem mostrados na prática, sendo o PE é um exemplo dessa dificuldade ${ }^{(6-8)}$.

A utilização da tecnologia da informação, como uma ferramenta de apoio ao processo de ensino aprendizagem na área da enfermagem, é recente, porém, diversas iniciativas têm contribuido para mudar esse quadro(4,11).

A atividade de criação pelo método de desenvolvimento visual demonstrou ser de fácil execução, pois o que era produzido na área de trabalho do software tinha imediata visualização, sem a necessidade de simulação em ambiente do navegador. Por meio desse método, a atividade de desenvolvimento foi mais rápida e efetiva, se comparada com 
o método de programação por códigos $\mathrm{HTML}^{(9)}$.

A forma de apresentação de um determinado conteúdo em página web é um assunto em constante discussão. Diversos fatores estão envolvidos no complexo processo que ocorre quando uma pessoa acessa Website. A maioria dos usuários não lê todo o conteúdo de uma página web. O fenômeno que acontece diante da tela do monitor é o escaneamento da informação que interessa à pessoa que a acessa $^{(3)}$.

Ao avaliar a qualidade do conteúdo didático-científico do site sob a perspectiva do usuário, é importante considerar que páginas extensas e que precisam de barra de rolagem lateral não são favoráveis quando se pretende obter uma boa frequência ao Website ${ }^{(6)}$. Entretanto, ao considerar o caráter educativo do WebCardio, optou-se pela apresentação do conteúdo em forma de seções com navegação página a página, a partir de ligações (hyperlinks) no seu rodapé, algo que induz o usuário a usar a barra de rolagem e a escanear o conteúdo das páginas mais extensas $^{(5)}$

\section{CONCLUSÃO}

A partir dos resultados descritos e das observações realizadas durante a elaboração do WebCardio, em suas fases de conceituação, de desenvolvimento e de implementação, conclui-se que: a primeira fase foi a que empreendeu o maior gasto de tempo, por exigir exaustiva pesquisa bibliográfica para a composição e elaboração de seu conteúdo teórico científico.

Nesse processo, a adoção do modelo de web site sugerido por Trochim foi de grande utilidade. Durante a fase de desenvolvimento, pode-se dizer que o trabalho de criação com o método de desenvolvimento visual foi uma atividade prazerosa e de grau fácil e que exigiu conhecimentos básicos de informática. A partir dessa conclusão, é possivel afirmar que a atividade pode ser executada por outros enfermeiros, seja qual for a área a ser abordada, basta ter um básico conhecimento de microinformática.

Sendo assim, os pesquisadores se propõem a atualizar o conteúdo deste Website periodicamente, sendo acrescido de novas informações relevantes ao assunto e/ou substituição das consideradas ultrapassadas.

\section{REFERÊNCIAS}

1. Rangel RF, Backes DS, Ilha S, Zamberlan C, Siqueira HCH, Costenaro RGS. Formação para o cuidado integral: percepção de docentes e discentes de enfermagem. Rev. Pesqui. Cuid. Fundam. 2017; 9(2): 488-94.

2. Silva RS, Almeida ARLP, Oliveira FA, Oliveira AS, Sampaio MRFB, Paixão GPN. Sistematização da assistência de enfermagem na perspectiva da equipe. Enferm. Foco. 2016; 7(2): 32-36.

3. Kobayashi RM, Leite MMJ. As competências tecnológicas no ensino de enfermagem cardiológica. Rev. Esc Enferm USP. 2015; 49(6): 971-77.

4. Prado C, Peres HHC, Leite MMJ. Tecnologia da informação e da comunicação em enfermagem. São Paulo: Atheneu; 2011.

5. Salvador PTCO, Martins CCF, Alves KYA, Pereira MS, Santos VEP, Tourinho FSV. Tecnologia no ensino de enfermagem. Rev. Baiana Enferm. 2015; 29(1): 33-41.

6. Martins MCT, Chianca TCM. Construção de um software com o processo de enfermagem em terapia intensiva. J. Healt Inform.

\section{6; 8(4): 119-25}

7. Góes FSN, Fonseca LMM, Camargo RAA, Hara CYN, Gobbi JD, Stabile AM. Elaboração de um ambiente digital na educação profissionalizante em enfermagem. Cienc. Enferm. 2015; 21(1): 8190.

8. Trochim WMK. Evaluating websites. New York: Cornell University 1996.

9. Long JD, Gannaway P, Ford C, Doumit R, Zeeni N, SukkariehHaraty $\mathrm{O}$, et al. Effectiveness of a technology-based intervention to teach evidence-based practice: the EBR tool. Worldviews Evid. Based. Nurs. 2016; 13(1): 59-63.

10. Votre VP. C++: explicado e aplicado. Rio de Janeiro: Alta Books Editora; 2016

11. Tibes CM, Dias JD, Westin UM, Domingues AN, ZemMascarenhas SH, Évora YDM. Desenvolvimento de recursos educacionais digitais para o ensino de enfermagem. Rev. Enferm. UFPE. 2017; 11(3): 1326-34. 